Musées, Patrimoine et Culture scientifiques et techniques

128 | 2010

mars - avril 2010

\title{
Promouvoir la culture scientifique et technique en Afrique
}

Promoting scientific and technical culture in Africa

\section{Marie-Lise Sabrié}

\section{OpenEdition Journals}

Édition électronique

URL : http://journals.openedition.org/ocim/159

DOI : 10.4000/ocim.159

ISSN : 2108-646X

\section{Éditeur}

OCIM

Édition imprimée

Date de publication : 1 mars 2010

Pagination : 5-11

ISSN : 0994-1908

Référence électronique

Marie-Lise Sabrié, « Promouvoir la culture scientifique et technique en Afrique », La Lettre de l'OCIM [En ligne], 128 | 2010, mis en ligne le 01 mars 2012, consulté le 19 avril 2019. URL : http:// journals.openedition.org/ocim/159; DOI : 10.4000/ocim.159 


\title{
Promouvoir la culture scientifique et technique en Afrique
}

\author{
Marie-Lise Sabrié *
}

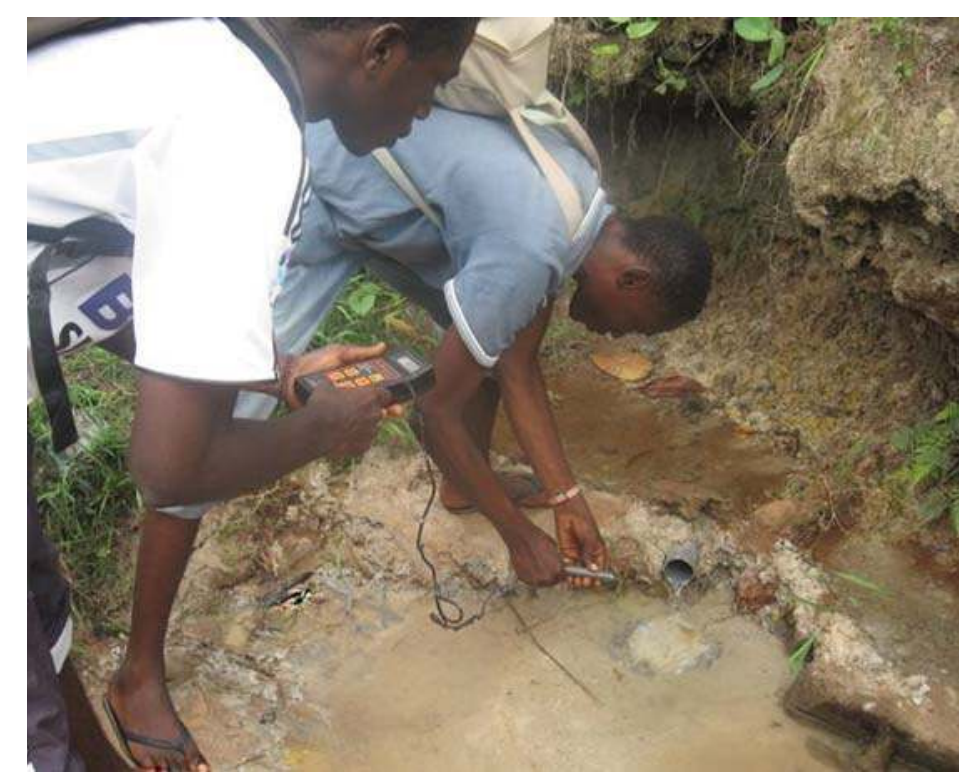

Atelier scientifique où de jeunes camerounais sont initiés à l'étude d'une zone humide proche de la capitale, Yaoundé () Association des Volontaires pour le Développement Socio-Économique, Cameroun

* Marie-Lise Sabrié est ingénieure de recherche, responsable du secteur Culture scientifique, délégation à l'Information et à la Communication, Institut de Recherche pour le Développement (IRD) marie-lise.sabrie@ird.fr
La mise en place par l'Institut de Recherche pour le Développement (IRD) d'un programme de promotion de la culture scientifique et technique dans dix pays africains a permis de mettre en évidence les attentes des populations mais également les faiblesses structurelles dans ce domaine : I'une des responsables de ce projet dresse le bilan de cette opération qui - malgré les difficultés constatées - laisse entrevoir des possibilités futures de développement pour les initiatives en faveur de la diffusion de la culture scientifique et technique en Afrique.

Fin 2003, le ministère des Affaires étrangères et européennes confie à l'IRD (1) la mise en œuvre d'un programme de promotion de la culture scientifique et technique (PCST) dans dix pays du continent africain, pour beaucoup francophones : Burkina Faso, Cameroun, Centrafrique, Djibouti, Madagascar, Mali, Maroc, Sénégal, Tchad, Yémen. Ce projet est doté d'un financement de plus de deux millions d'euros par le Fonds de solidarité prioritaire mis en place par la Direction générale de la Coopération internationale et du Développement. Il s'agit de la première initiative de cette ampleur destinée à promouvoir la culture scientifique dans un pays du Maghreb et en Afrique subsaharienne. Pendant près de cinq ans, ce programme d'actions, ambitieux et novateur, va révéler au sein de la société civile africaine une dynamique forte et souvent insoupçonnée en faveur d'une meilleure diffusion des savoirs scientifiques et 
techniques. Alors que le PCST vient de s'achever, cet article tire le bilan de ces années d'expérience par l'équipe coordinatrice à l'IRD (2).

\section{Un fossé entre sciences et sociétés}

Le lancement du programme PCST s'est fondé sur le constat de la profonde fracture qui existe entre la science et la société en Afrique subsaharienne, tel que le décrit sans ambiguité le sociologue camerounais Jean-Marc Ela : «La Science est loin d'être au centre des préoccupations actuelles des sociétés africaines (...). Dans la tourmente qui frappe le continent noir, les nouvelles générations doutent de l'utilité même de la science. Elles se demandent à quoi elle sert et si elle n'est pas un luxe face aux urgences de survie au quotidien. (...) Au-delà des laboratoires et des campus, il faut bien reconnaître que l'image de la science et de la recherche pose un problème fondamental en Afrique bien plus qu'ailleurs. À l'évidence, cette image est bien floue, voire effacée. (...) Tout donne l'impression qu'on peut parfaitement s'en [des scientifiques] passer dans la mesure même où l'on ne sait pas très bien ce qu'ils représentent » (3).

De fait, force est d'observer que, sur le continent africain, les populations ont difficilement accès aux savoirs scientifiques. Ceci s'explique par un certain nombre de facteurs. La plupart des pays en Afrique subsaharienne sont dépourvus d'institutions pérennes dédiées à la diffusion de la culture scientifique et technique (de type muséum, musée ou centre de science). De plus, il n'y a que peu d'associations spécialisées dans ce domaine; et, quand ces structures existent, elles manquent de moyens, d'expérience et de soutien institutionnel. Par ailleurs, les producteurs de sciences ou de technologies que sont les universités ou les organismes de recherche se lancent rarement dans des actions de communication vers le grand public. De surcroît, les médias (journaux, chaînes de radio ou télévision, y compris Internet) apparaissent peu comme des vecteurs d'information scientifique auprès des populations, et les revues de vulgarisation sont quasi inexistantes. Enfin, même en faisant abstraction des taux d'analphabétisme plus élevés qu'ailleurs sur le continent africain, l'école ne s'affirme pas comme le moyen privilégié de transmission des connaissances scientifiques qu'elle devrait être. Souvent cantonnée à des enseignements académiques, en sureffectif et dépourvue de ressources pédagogiques, elle ne semble pas donner le goût et le plaisir des sciences. Comme dans les pays développés, beaucoup font le constat d'une désaffection des élèves du secondaire et des étudiants à l'université pour les filières scientifiques et techniques. C'est le cas par exemple au Sénégal où à peine $35 \%$ des élèves de Terminale sont inscrits dans un cursus scientifique, avec des taux bien inférieurs pour les jeunes filles et dans les universités. Ainsi, pour toutes ces raisons, par manque de médiateurs et d'outils de médiation, un important fossé existe dans cette partie du monde entre la science et la société, entre une élite qui y a accès et ceux très nombreux qui ne peuvent bénéficier des connaissances qu'elle produit.

\section{Susciter une dynamique nouvelle}

Dans un tel contexte, l'IRD avec le programme PCST s'est fixé plusieurs objectifs majeurs :

- susciter, dans les dix pays concernés, des capacités de production et de diffusion de la culture scientifique ;

- professionnaliser les acteurs ;

- fédérer les initiatives ;

- mobiliser les décideurs ;

- offrir une visibilité aux acteurs et à leurs projets.

L'un des premiers champs d'actions privilégiés a été d'encourager la réalisation de projets portés par des structures nationales et sélectionnés par un comité scientifique. Les sept appels à projets lancés de 2004 à 2008 ont vu une très forte mobilisation avec plus de 561 projets soumis par 290 structures candidates différentes. Certes, l'élan a été inégal selon les pays, le Maroc et le Cameroun se distinguant

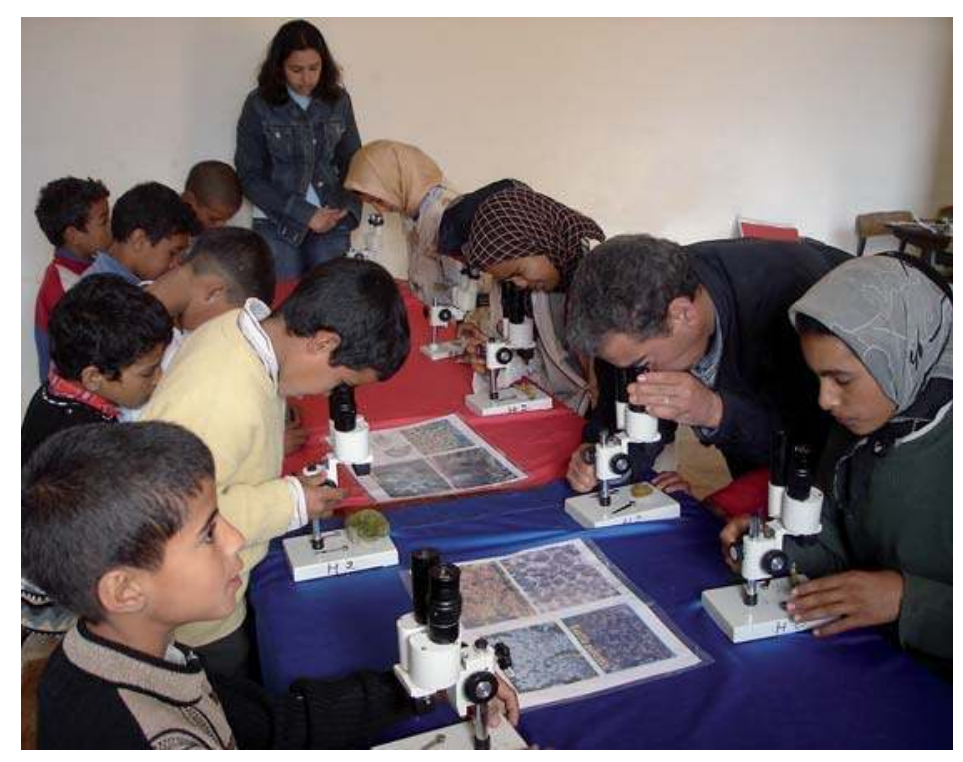

Étude de lichens, bio-indicateurs de la qualité de l'air, dans le cadre d'un club de sciences au Maroc (c) AESVT Safi, Maroc 
nettement par le nombre de candidatures (respectivement $25 \%$ du total), suivis par le Sénégal et le Burkina Faso (18 et $11 \%$ ). Après sélection, plus de 150 projets ont bénéficié d'un soutien financier et technique, avec une dotation de 9000 euros en moyenne par projet, ce qui a représenté près de $60 \%$ du budget dévolu au programme (4). Ainsi, malgré le sombre panorama esquissé plus haut, la mise en œuvre du PCST a mis en lumière une dynamique forte et nouvelle en Afrique francophone en faveur de la culture scientifique et technique.

La mobilisation créée par les appels à projets a été avant tout le fait d'associations ou d'ONG qui ont représenté près des deux tiers des lauréats, contre à peine $20 \%$ pour les universités, les laboratoires et les organismes de recherche. L'un des acquis majeurs de ce programme est alors d'avoir incité des associations à s'instituer, souvent pour la première fois, comme passeurs de science et médiateurs entre le monde de la recherche et la société civile. Selon différentes études confiées au CERLIS (5), les porteurs de projets sont le plus souvent des structures traditionnellement engagées dans des actions de développement, d'éducation ou des activités culturelles, mais souvent néophytes dans le domaine de la culture scientifique et technique.

\section{Les sciences réappropriées}

Les activités de ces structures frappent par leur grande diversité : expositions, clubs de sciences, conférences et débats, émissions radiophoniques, pièces de théâtre, bandes dessinées, sorties découvertes... Pour près de la moitié, elles ont été mises en œuvre dans le cadre de festivals scientifiques ou semaines thématiques (astronomie, environnement, santé), associant de multiples formes d'action. C'est le cas, par exemple, de la manifestation Scientifika au Sénégal, des deux éditions du FESTY, le festival des sciences et des techniques de Yaoundé, ou encore des caravanes des sciences en Centrafrique, au Cameroun et au Maroc. Par ailleurs, les clubs de sciences ont représenté plus d'un tiers des projets. D’une part parce que ce type d'activité répond bien aux attentes des jeunes, public prioritaire pour la quasi-totalité des structures quel que soit le pays. D'autre part, parce qu'un grand nombre de projets financés dans le cadre du PCST ont été mis en œuvre en lien avec des établissements scolaires, plus familiers que d'autres structures de la démarche pédagogique initiée par des clubs scientifiques. Enfin, il est à noter que près de $15 \%$ des projets ont eu recours au spectacle vivant, mode d'expression
Ateliers de chimie
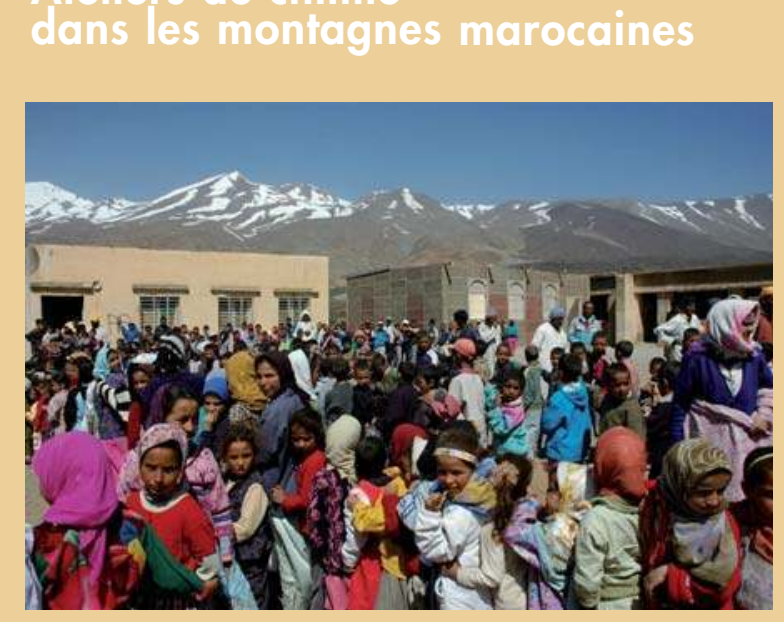

Dans des villages isolés du Maroc, une foule d'enfants attend patiemment de participer à des ateliers de chimie. () Association Les Rangs d'Honneur, Maroc

Le Maroc apparaît comme l'un des pays les plus dynamiques parmi ceux concernés par le programme PCST : sur 157 projets lauréats des appels à projets, une cinquantaine sont marocains! Clubs scientifiques, expositions, festival de films scientifiques, conférences, visites de laboratoires, sorties de terrain... de Rabat à Oujda, de Tanger à Marrakech, dans les écoles, les lycées, les universités, les places publiques et même dans les souks... des opérations de diffusion de la culture scientifique et technique se multiplient. Un projet mené par les Rangs d'Honneur offre un bel exemple de ces initiatives. Cette association organise des ateliers scientifiques pour les enfants dans le cadre de campagnes de consultations médicales gratuites se déplaçant de village en village dans des régions de montagne enclavées. Ces ateliers itinérants constituent une réponse particulièrement intéressante au dénuement en matière d'équipements de culture scientifique. Encadrées par une enseignante chercheuse de l'université de Casablanca et traduites en berbère, ces animations sur la pollution de l'eau ou les carences en iode, associant expérimentations et manipulations, ont rassemblé plus de 1500 enfants, dépassant largement les prévisions initiales.

(www.latitudesciences.ird.fr/realisations/rangsdhonneur/index.htm) 
généralement peu usité dans le domaine de la culture scientifique. Ce choix s'explique sans aucun doute par le fait que les pièces de théâtre en langue locale permettent de toucher un public analphabète ou maîtrisant mal le français et/ou la langue officielle, mais aussi parce qu'elles s'inscrivent dans une tradition orale encore très vivante pour nombre de populations en Afrique subsaharienne, en particulier en milieu rural.

Au cœur des projets, les thématiques abordées ont été multiples : l'astronomie, la biodiversité, les maladies infectieuses, l'informatique, l’archéologie, les énergies renouvelables, les ressources en eau, pour n'en citer que quelques-unes. Alors que les activités destinées à initier aux disciplines fondamentales comme les mathématiques ou la physique ont été relativement peu nombreuses, on a pu observer une dominante majeure avec deux thèmes - l'environnement et la santé - privilégiant des sciences plus appliquées.

Les projets sont apparus très en phase avec les problématiques auxquelles sont confrontées les populations dans les pays du programme : déforestation, pollution de l'eau, santé des femmes, paludisme, sida... Lors du colloque organisé à Marseille en septembre 2009 pour la clôture du programme (6), les intervenants ont souligné combien, en Afrique subsaharienne, l'appropriation des connaissances scientifiques nécessitait une "reformulation » des problématiques abordées à l'aide de représentations culturelles familières du public et en lien avec ses préoccupations quotidiennes. À cette occasion, le rôle des médiateurs locaux a été mis en exergue du fait de leur capacité à favoriser une réappropriation des questions scientifiques en les inscrivant en perspective avec la culture et l'environnement des populations. Comme l'a affirmé un animateur camerounais, face au désengagement des institutions (scolaires, universitaires ou scientifiques), "les associations sont une alternative sérieuse et efficace pour la promotion des sciences et techniques, car elles réussissent à mettre en miroir le savoir scientifique et le savoir endogène».

\section{Se professionnaliser}

Les enquêtes réalisées par le CERLIS dans les pays où a été mis en œuvre le PCST ont souligné le peu d'expérience des différentes structures en matière de diffusion de la culture scientifique et ont fait état de leur forte attente en matière de formation. Pour répondre à ce besoin de professionnalisation, le programme a organisé 14 sessions régionales de formation à la

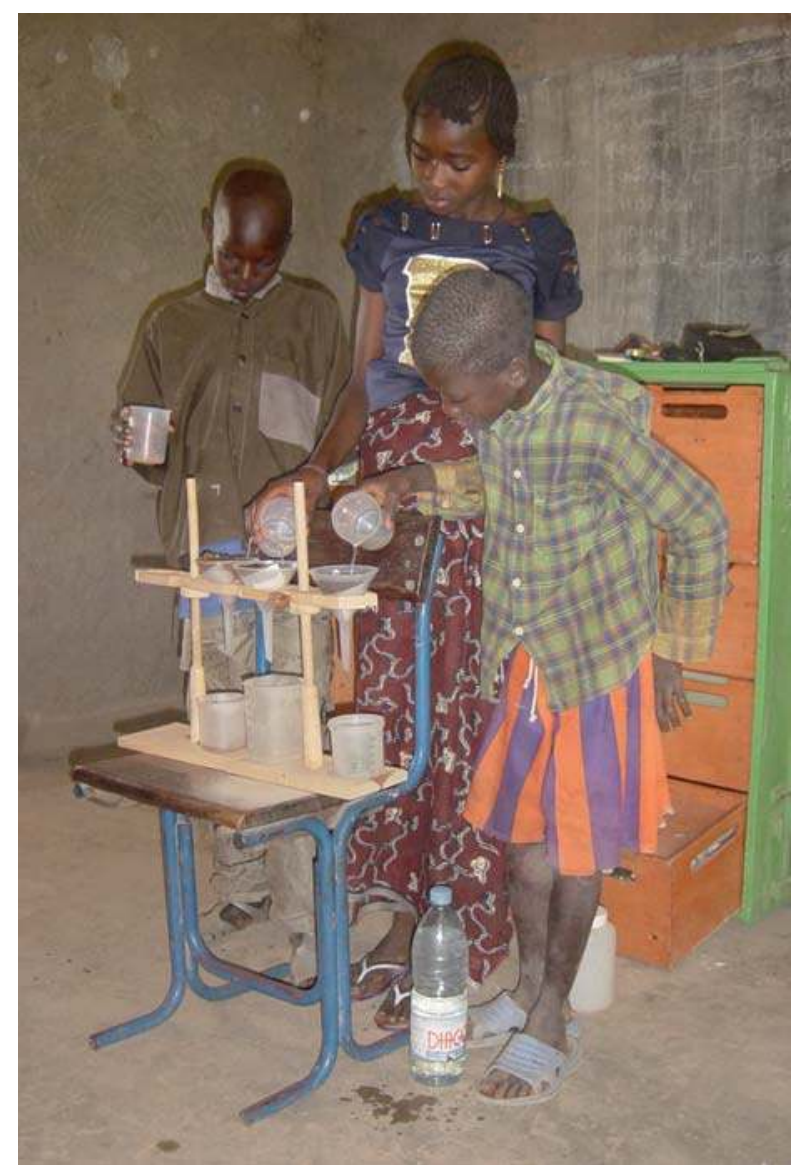

Cette malle scientifique sur les plantes et les sols a fait l'objet de nombreuses animations dans des écoles rurales du Burkina Faso. (c) Association Karamba Toure, Mali

gestion de projets, à l'animation ou à la communication scientifiques au Burkina Faso, au Sénégal et au Maroc ; celles-ci ont bénéficié à 250 personnes, membres d'associations, animateurs, enseignants ou chercheurs. Ces formations ont été confiées à des experts en médiation scientifique comme l'association Planète Sciences, le Palais de la Découverte, le CCSTI Centre Sciences ou encore le réseau des CPIE (centres permanents d'initiatives pour l'environnement). Afin d'élargir le nombre de bénéficiaires et prolonger ces sessions de formation, Les carnets de route de la culture scientifique, trois guides pratiques destinés à tous ceux souhaitant s'initier à la réalisation d'activités de culture scientifique et technique, ont été édités par l'IRD et diffusés à plus de 3000 exemplaires. Ces outils sont également téléchargeables sur le site Internet Latitudesciences accompagnés d'autres ressources : un lexique (en partenariat avec la Cité des Sciences et de l'Industrie), des dossiers d'information thématiques, une banque d'images scientifiques ${ }^{(7)}$... Ces différentes actions de formation et ces outils pédagogiques, 
au-delà de leurs bénéfices immédiats pour de très nombreux acteurs, ont créé un "pool » de compétences. Ceci a constitué l'un des acquis importants du programme en offrant le gage d'une pérennisation des actions - et des actions de qualité - dans les pays, ainsi que l'a souligné le directeur d'une association marocaine : "pérenniser nos activités consiste d'abord à nous professionnaliser, car la culture scientifique et technique est un travail difficile et de longue haleine».

\section{Créer des synergies}

Dans nombre de pays, lorsque le PCST a été lancé, la dynamique en faveur de la culture scientifique qui semblait s'amorcer n'était pas seulement contrecarrée par le manque de professionnalisme mais également par la dispersion des structures. "Nous avions souvent les mêmes objectifs, développions des activités semblables et sur les mêmes sujets, sans même nous connaître faute d'avoir eu des occasions de nous rencontrer », soulignait ainsi l'un des lauréats des appels à projets. La faible concertation des acteurs et l'insuffisante cohésion de leurs projets trouvent en grande partie leur explication dans l'absence, au moment du lancement du programme, de politiques nationales en faveur de la culture scientifique, susceptibles de tisser des liens entre les structures et d'initier des projets communs. Le programme PCST a eu alors pour ambition de créer une plus grande synergie, notamment par l'organisation de rencontres offrant l'occasion de fructueux échanges d'expériences. Dans cette perspective, cinq séminaires ont été organisés au Maroc, au Burkina Faso, au Cameroun et en France, auxquels ont participé au total plus de 400 personnes, parmi les principaux acteurs impliqués dans la diffusion de la culture scientifique et technique dans les pays du programme ${ }^{(8)}$.

Ces rencontres ont eu pour effet de susciter la création de réseaux, en particulier au Maroc et au Cameroun. Ces réseaux ont offert aux structures la possibilité de se fédérer et de mutualiser leurs moyens pour l'élaboration de projets communs. C'est le cas notamment au Cameroun avec le COPCST (collectif des organisations de promotion de la culture scientifique et technique) qui, en réunissant une quinzaine d'associations, a été à l'origine de la première Fête de la Science au Cameroun - le FESTY. Il en est aujourd'hui à sa deuxième édition et bénéficie du soutien des ministères en charge de la Recherche et de l'Éducation nationale. L'autre bénéfice de ces actions collectives est d'avoir permis aux structures de gagner en reconnaissance auprès des pouvoirs publics et de leur avoir donné plus de poids pour sensibiliser ces derniers aux enjeux de la culture scientifique. Le réseau marocain - le RNCST, réseau national de culture scientifique et technique - est, de ce point de vue, exemplaire. Créé en 2008, il réunit 6 universités marocaines et couvre l'ensemble du pays. Suite à un colloque organisé par le RNSCT à Rabat en octobre 2009, le ministère marocain de l'Enseignement supérieur a chargé ce réseau d'élaborer des propositions pour organiser le paysage de la culture scientifique au Maroc ${ }^{(9)}$.

\section{Visibilité}

L'absence de visibilité des structures et de leurs activités, en particulier dans les médias d'Afrique francophone où la science est réduite à la portion congrue, est apparue comme une lacune majeure. Le programme PCST a alors beaucoup misé sur la communication. Il s'agissait de favoriser l'échange d'informations entre les acteurs mais aussi de rendre plus

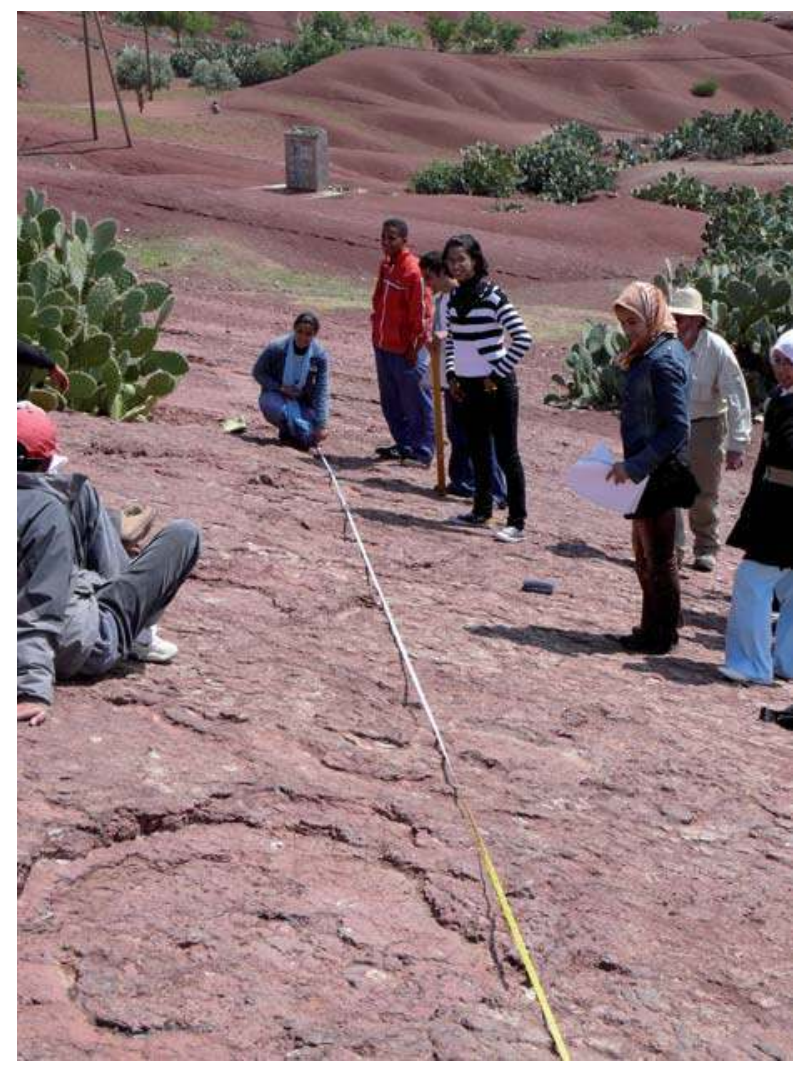

Initiation de lycéens marocains aux techniques de recherche de terrain en Paléontologie dans le cadre du projet "Au pays des dinosaures» (c) AESVT Demnate, Maroc 
visibles leurs actions. Dans cette perspective, Latitudesciences, le premier site Internet francophone dédié aux acteurs de la culture scientifique sur le continent africain, a été créé ${ }^{(4)}$. Il permet à chaque porteur de projet de présenter sa structure et les activités mises en œuvre. Des rubriques informent également sur toutes les manifestations ouvertes au grand public ou recensent près de 400 sites utiles pour la mise en œuvre de projets de culture scientifique.

Par ailleurs, pour renforcer la visibilité des projets et, plus généralement celle de la culture scientifique dans les pays du programme, les structures ont été encouragées à communiquer sur leurs actions auprès des médias. Cette stratégie de communication a généré près de 350 retombées presse, essentiellement dans la presse écrite, à la radio et sur Internet. Cellesci sont le résultat d'une mobilisation de la presse par les porteurs de projets mais aussi d'un partenariat établi par l'IRD avec l'agence Syfia International, qui a effectué de nombreux reportages sur les activités mises en œuvre dans le cadre du PCST.
Enfin, le PCST a particulièrement encouragé les manifestations propices à rassembler un large public et constituant une vitrine pour la culture scientifique. Les meilleurs exemples en sont les festivals de science qui, comme au Maroc (Fête de la science), au Burkina Faso (Forum de la recherche scientifique et technologique), en République Centrafricaine (Caravane des Sciences) ou à Madagascar (Quinzaine scientifique), investissent des centres culturels, les campus des universités ou même les places publiques, attirant souvent plusieurs milliers de visiteurs. Grâce à un fort partenariat institutionnel (ministères, collectivités locales, universités, organismes de recherche), le programme a également organisé la tournée d'une exposition Sciences au Sud-consacrée aux enjeux de la recherche scientifique pour le développement du Sud. De l'Afrique du Sud au Maroc, présentée sur des places ou des jardins publics de grandes villes (Antananarivo, Bamako, Ouagadougou, Niamey, Dakar, Rabat, Marrakech, N'djamena, Yaoundé), cette exposition a reçu plus de 60000 visites guidées

\section{Savonte comédie en pays Lobi}

Comment sensibiliser aux avancées des sciences les populations des régions rurales africaines où le taux d'analphabétisme peut atteindre plus de $80 \%$ ? Une association burkinabé - l'association pour la promotion des femmes de Gaoua (APFG) - a relevé le défi dans des villages de l'ouest du Burkina Faso. Celleci s'est entourée des conseils d'experts (démographes, nutritionnistes, médecins) pour réaliser une campagne d'information sur la santé des femmes et la nutrition des enfants, en mettant au cœur du dispositif le théâtre forum scientifique. Les saynètes jouées en langue locale par des femmes de l'association mettent face à face les savoirs issus de la science et les pratiques traditionnelles. À l'issue des représentations qui attirent en moyenne 200 personnes, le public est encouragé à intervenir pour donner son point de vue et débattre des messages transmis. Le théâtre forum ouvre alors un surprenant dialogue entre science et société. Pour Ini Damien, responsable de l'association, cette méthode participative de transmission des savoirs a été très enrichissante pour l'ensemble de la communauté, permettant une véritable appropriation des savoirs scientifiques : «ces projets, inspirés de notre réalité, sont partis de nous-mêmes. C'est le facteur de leur réussite : aujourd'hui, les femmes de nos villages possèdent ces connaissances scientifiques et personne ne peut plus le leur reprendre ». Un court-métrage, consultable sur Internet, retrace cette expérience originale (www.ird.fr, rubrique La médiathèque/ canal IRD).

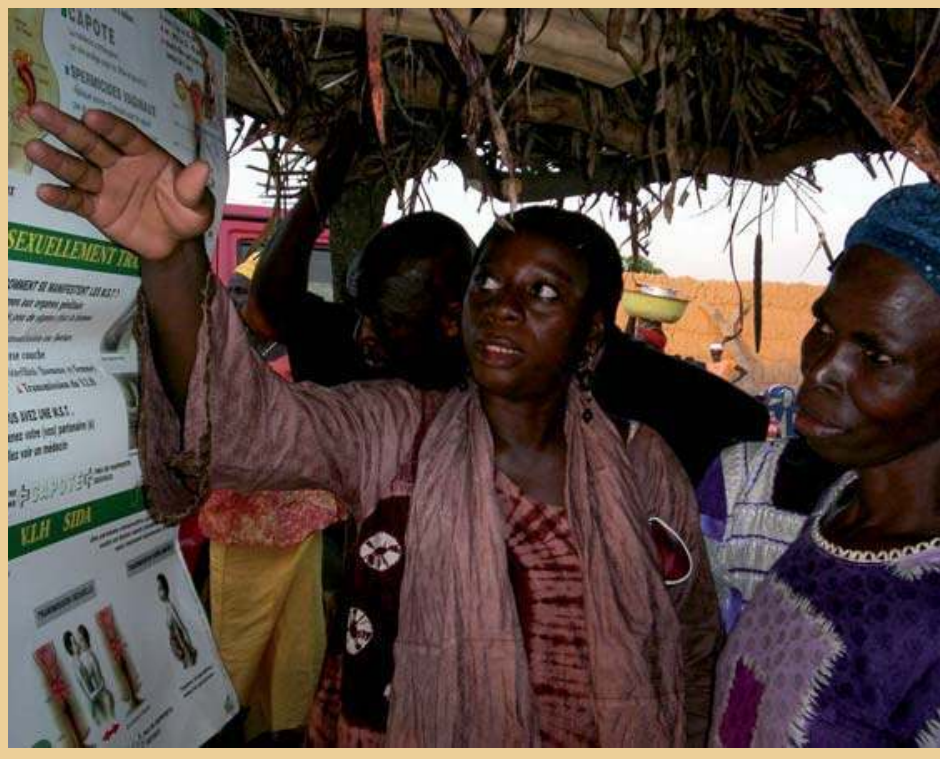

Campagne d'information sur les maladies sexuellement transmissibles auprès de villageoises du Burkina Faso par l'APFG () APFG, Burkina Faso 


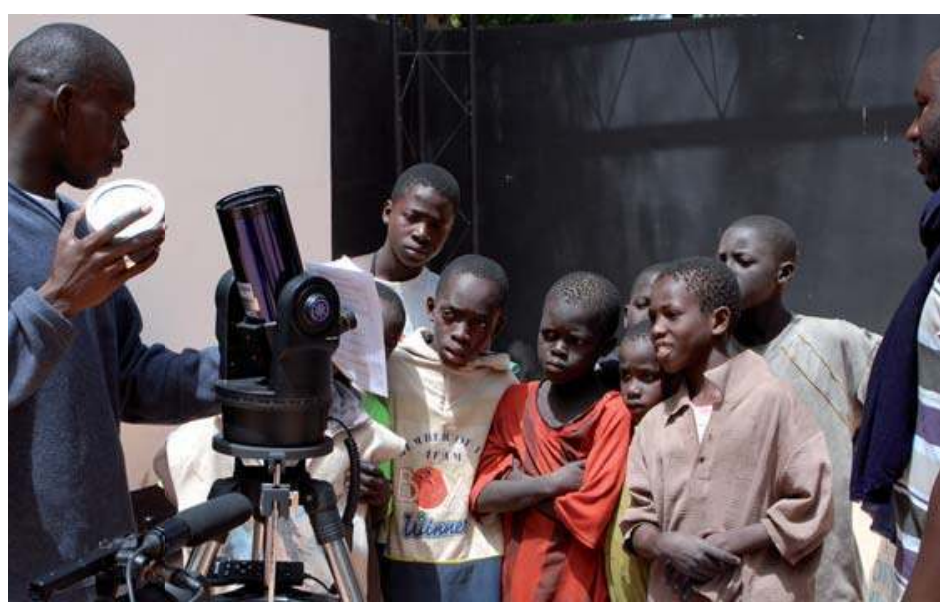

Observation du soleil lors du festival d'astronomie Saint Louis sous les étoiles qui a associé au Sénégal l'université Gaston Berger de Saint-Louis, le Centre culturel français Jean-Mermoz, l'association sénégalaise pour la promotion de l'Astronomie et le Palais de la Découverte () IRD/Raphaële Nisin

auxquelles se sont ajoutées les visites libres. En s’installant dans des espaces urbains très fréquentés, elle a montré, s'il le fallait, qu'il existe bien un public pour la culture scientifique en Afrique, et combien «l'homme de la rue » sur ce continent est curieux de sciences et avide des innovations qu'elles produisent.

Comment expliquer un accueil aussi favorable du public, et notamment des jeunes, observé pour nombre de manifestations organisées dans le cadre du PCST ? En l'absence d'enquête auprès des populations, il est difficile d'apporter des réponses précises à cette question. Cependant, parmi les quelques hypothèses qui pourraient être esquissées, figure en bonne place la présentation de spectacles, animations ou même d'expositions par des médiateurs s'exprimant dans la langue maternelle du public. Dans des pays où les sciences et les techniques se disent ou s'écrivent habituellement en langue "étrangère ", en l'occurrence le français ou l'anglais - notamment à l'école ou à l'université -, ceci permet de lever les barrières linguistiques mais aussi celle de l'analphabétisme, comme l'explique l'organisateur d'une exposition scientifique à Ouagadougou : " une importante population analphabète y a trouvé son compte puisque les animateurs guidaient les gens ; ils parlaient trois langues, le dioula, le moore et la langue française. Donc la communication passait » $(10)$. Si, très certainement, de nombreux autres facteurs ont favorisé le succès populaire de ces projets de culture scientifique, une telle médiation a contribué à rendre plus familières les sciences et les techniques à ces populations du continent africain.

\section{Conclusion}

La mise en œuvre du programme PCST a contribué à souligner les nombreuses difficultés auxquelles se heurte la diffusion de la culture scientifique sur le continent africain : le faible soutien des pouvoirs publics, une communauté scientifique peu encline à communiquer avec le grand public, un enseignement des sciences trop académique, la quasi-absence des sciences et des technologies dans les médias africains, les moyens très réduits dont disposent les structures tant en termes financiers ou logistiques qu'en matière de ressources humaines ou d'accès à l'information. Cependant, la forte dynamique qui se fait jour actuellement au sein de la société civile africaine montre qu'une politique de soutien aux initiatives et aux projets, telle qu'initiée avec le PCST par le ministère des Affaires étrangères et européennes et l'IRD, mériterait d'être poursuivie par des instances nationales mais aussi par la coopération internationale, encore trop peu présente sur ce terrain.

L'auteur remercie Raphaële Nisin et Marie-Ève Migueres pour leur relecture et leurs suggestions sur cet article.

\section{Notes}

(1) L'Institut de Recherche pour le Développement (ex-Orstom) développe des programmes de recherche pluridisciplinaires dans les pays du Sud. Aux côtés de ses activités scientifiques, l'IRD a une mission de diffusion de l'information scientifique et technique en France et dans ses pays partenaires. (2) Cette équipe a été animée par Raphaële Nisin, puis Marie-Ève Migueres et Justine Bonne, sous la direction de Marie-Lise Sabrié ; au ministère des Affaires étrangères et européennes : Yves de la Croix et Sylvie Ballet.

(3) Ela, J.-M. Les cultures africaines dans le champ de la rationalité scientifique. Paris : L'Harmattan, 2008, 210 p.

(4) Voir le site www.latitudesciences.ird.fr où l'analyse complète des appels à projets et une présentation des structures lauréates sont disponibles.

(5) Ces études réalisées par Séverine Dessajan et Elsa Ramos du Centre de Recherche sur les Liens Sociaux (CERLIS, UMR 8070 CNRS/université Paris V) sont téléchargeables sur le site Internet www.latitudesciences.ird.fr, rubriques PCST/études et articles.

(6) Séminaire international La culture scientifique au Sud: enjeux et perspectives pour l'Afrique, dont les actes sont disponibles sur www.latitudesciences.ird.fr

(7) www.latitudesciences.ird.fr, rubrique Outils

(8) Les comptes rendus de ces séminaires sont disponibles sur www.latitudesciences.ird.fr, rubrique PCST-les actions.

(9) La Culture Scientifique et Technique au Maroc: esquisse d'un état des lieux et perspectives d'une politique nationale à la lumière des expériences étrangères, siège du CNRST, Rabat, 26-28 octobre 2009.

(10) Étude menée par Séverine Dessajan et Elsa Ramos au Burkina Faso. 